\title{
The pancreatic stellate cell: a star on the rise in pancreatic diseases
}

\author{
M. Bishr Omary, ${ }^{1,2}$ Aurelia Lugea, ${ }^{3}$ Anson W. Lowe, ${ }^{1}$ and Stephen J. Pandol ${ }^{3}$
}

1Department of Medicine, VA Palo Alto Health Care System, Palo Alto, California, USA. ${ }^{2}$ Stanford University School of Medicine, Stanford, California, USA. 3USC-UCLA Research Center for Alcoholic Liver and Pancreatic Diseases and Veterans Affairs Greater Los Angeles Healthcare System, Los Angeles, California, USA.

\begin{abstract}
Pancreatic stellate cells (PaSCs) are myofibroblast-like cells found in the areas of the pancreas that have exocrine function. PaSCs are regulated by autocrine and paracrine stimuli and share many features with their hepatic counterparts, studies of which have helped further our understanding of PaSC biology. Activation of PaSCs induces them to proliferate, to migrate to sites of tissue damage, to contract and possibly phagocytose, and to synthesize ECM components to promote tissue repair. Sustained activation of PaSCs has an increasingly appreciated role in the fibrosis that is associated with chronic pancreatitis and with pancreatic cancer. Therefore, understanding the biology of PaSCs offers potential therapeutic targets for the treatment and prevention of these diseases.
\end{abstract}

\section{Introduction}

The pancreas can be functionally divided into an exocrine component, which produces enzymes that aid digestion in the gastrointestinal tract, and an endocrine component composed of the islets, which, among other functions, secretes hormones that regulate carbohydrate metabolism. The exocrine component consists primarily of clusters of acinar cells (known as acini) that secrete digestive enzymes into the lumen of the acini. These acini drain the digestive enzymes into the duodenum through the pancreatic ducts (Figure 1). Pancreatic stellate cells (PaSCs) are one of several resident cells in the exocrine pancreas. They are present in the periacinar space and have long cytoplasmic processes that encircle the base of the acinus (Figure 1 and Figure 2, A and B). They can also be found in perivascular and periductal regions of the pancreas (1-4) and serve as key participants in the pathobiology of the major disorders of the exocrine pancreas, including chronic pancreatitis and pancreatic cancer. In these disorders, PaSCs participate in disease pathogenesis after transforming from a quiescent state into an "activated" state (also known as a "myofibroblastic" state).

Approximately $70 \%-90 \%$ of cases of chronic pancreatitis result from alcohol abuse, while the remainder of cases are associated with genetic disorders (for example, hereditary pancreatitis and cystic fibrosis) and unknown causes (for example, idiopathic pancreatitis) (5-8). The course of chronic pancreatitis is characterized by recurrent episodes of acute pancreatitis, which cause parenchymal injury and necrosis, with increasing amounts of fibrosis, chronic inflammation, and parenchymal cell loss with each successive episode. Parenchymal cells in both the exocrine and, to a lesser extent, the endocrine pancreas are lost, and this leads to irreversible and debilitating exocrine, and ultimately endocrine, insufficiency that can be accompanied by a severe chronic pain syndrome. This series of events, which was determined by examination of human pancreatic tissue during alcohol-induced acute and chronic pancreatitis, has been termed the "necrosis-fibrosis sequence" and provides a framework for understanding chronic

Nonstandard abbreviations used: CTGF, connective tissue growth factor; GFAP, glial fibrillary acidic protein; HSC, hepatic stellate cell; PaSC, pancreatic stellate cell; TIMP-1, tissue inhibitor of metalloproteinase 1.

Conflict of interest: The authors have declared that no conflict of interest exists. Citation for this article: J. Clin. Invest. 117:50-59 (2007). doi:10.1172/JCI30082. pancreatitis (9). Adding to the morbidity and mortality of this disorder is the fact that patients with chronic pancreatitis have a substantially increased risk of developing pancreatic cancer $(10,11)$. Like chronic pancreatitis, adenocarcinoma of the pancreas, which is the most common form of pancreatic cancer, has a remarkable fibrotic component (12-15).

Several excellent reviews have covered the molecular and cellular regulation of pancreatic (16-18) and hepatic (19-22) fibrosis. However, here we consider the important role of the PaSC in the pathogenesis of key disorders of the pancreas. This Review will provide a synopsis of the properties and activation of PaSCs, as well as outline their role in pancreatic inflammation and cancer. We also compare PaSCs with their hepatic counterparts and describe potential PaSC regulatory pathways that might be used to therapeutic advantage.

\section{Properties of PaSCs}

Early studies of PaSCs capitalized on the knowledge and experience gained from study of their hepatic counterparts, the hepatic stellate cells (HSCs), which were first described by Karl von Kupffer in 1876 as Sternzellen (star cells) and initially felt to be phagocytic endothelial cells (reviewed in ref. 21). Stellate cells derive their name from their shape (stella in Latin means "a star") (Figure 1) and are also present in several other organs, including the kidney (23) and lung (24). Cells in the pancreas that were similar to HSCs in that they were fat-storing cells were first observed with the use of autofluorescence and electron microscopy in 1982 (1). They were identified when rats were given vitamin A, because cells with cytoplasmic fat droplets, such as HSCs (reviewed in ref. 25), become autofluorescent when vitamin A accumulates in these droplets (1). Subsequently, 2 landmark reports described the isolation and initial characterization of what have henceforth been termed PaSCs $(3,4)$. PaSCs express the intermediate filament proteins desmin and glial fibrillary acidic protein (GFAP) (Figure 2, A and B), which, together with the presence of intracellular fat droplets, serve to distinguish PaSCs from normal fibroblasts $(3,4)$ (Table 1 ).

PaSCs express intermediate filament proteins that usually characterize several cell types - for example, desmin, which characterizes myocytes; GFAP, which characterizes astrocytes; vimentin, which characterizes cells such as leukocytes, fibroblasts, and endothelial cells; and nestin, which characterizes neuroepithelial 


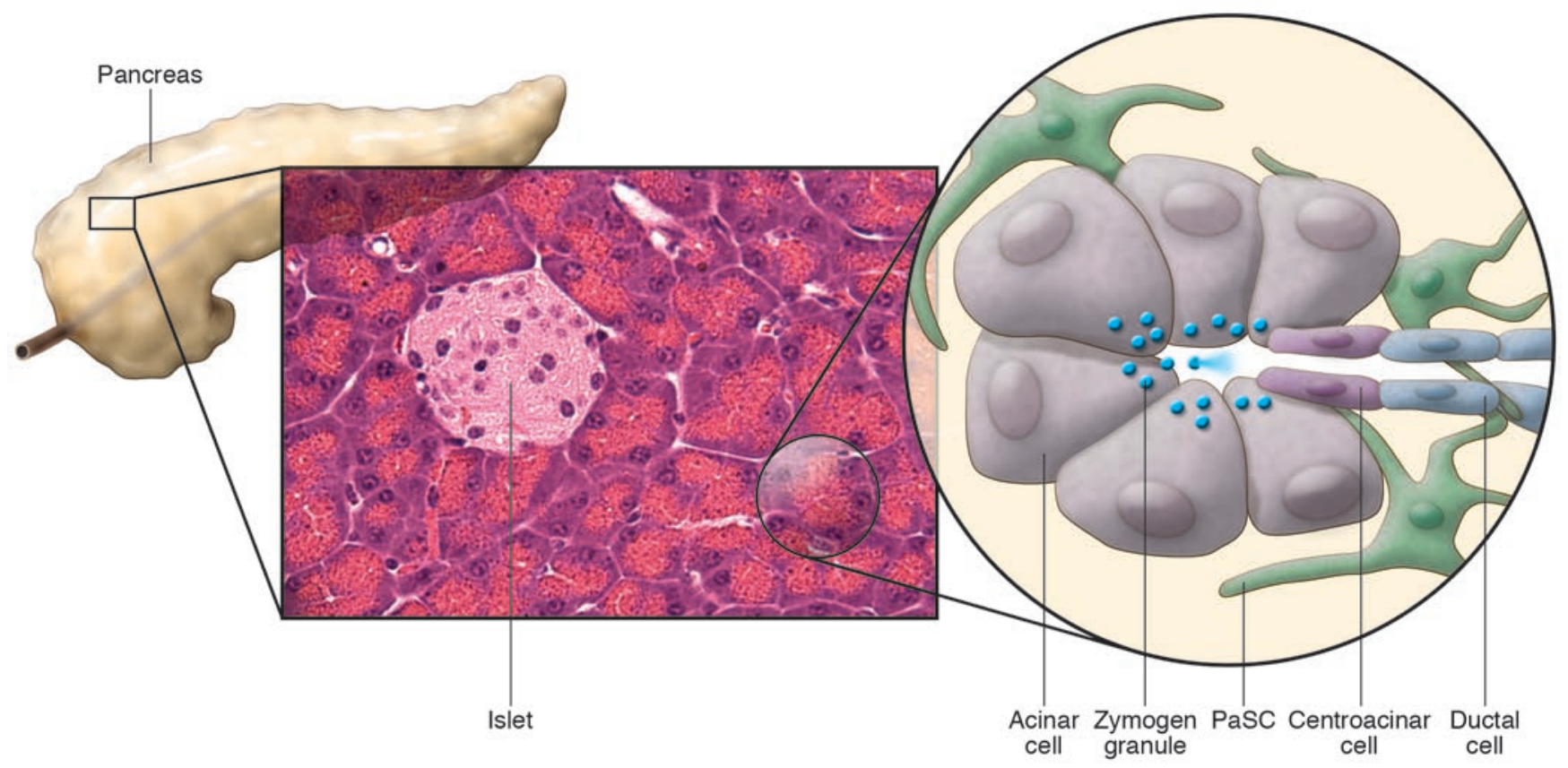

Figure 1

Schematic of the cellular components of the exocrine pancreas. The pancreas can be functionally divided into 2 components that are interspersed: an exocrine component that consists primarily of acini - clusters of acinar cells that feed into ductules — and an endocrine component composed of the islets. In the normal pancreas, quiescent PaSCs are present in the periacinar space. These cells have long cytoplasmic processes that encircle the base of the acinus. Zymogen granules release their contents of digestive enzymes into the pancreatic ductal system upon stimulation.

stem cells (26). The expression of such a diversity of intermediate filament proteins highlights that PaSCs have a broad range of potential properties, including contractility, the presence of cellular extensions to sense their environment, the potential to elaborate ECM components, and the potential to proliferate. However, it is important to note that these markers have clear limitations (see below) and that there are species differences (Table 1). Activation of quiescent PaSCs, which occurs when primary PaSCs are cultured and in the pancreas as a consequence of pancreatic injury, is associated with several morphologic changes $(3,4)$, including nuclear enlargement and enhanced prominence of the ER network (Table 1). Furthermore, in situ hybridization and immunohistochemical studies indicated that activated PaSCs express $\alpha$-SMA (also known as ACTA2) (Figure 2, C-E) and collagen type I, therefore marking these cells as a source of fibrosis in chronic pancreatitis and pancreatic adenocarcinoma $(14,27,28)$.

Initial efforts to isolate PaSCs produced cells that expressed $\alpha$-SMA and collagen I, collagen III, and collagen IV (29). However, use of the isolation protocols used in HSC studies, in particular density gradient centrifugation, allowed the isolation of quiescent PaSCs $(3,4)$. Isolated PaSCs are characterized as quiescent by the presence of desmin, GFAP, and intracellular fat droplets, but the absence of $\alpha$-SMA (Table 1). Primary PaSCs become activated during culture and attain a myofibroblast-like phenotype characterized by the disappearance of intracellular fat droplets, and the expression of $\alpha$-SMA and ECM proteins (collagen I, collagen III, and fibronectin) $(3,4)$ (Table 1). PaSCs also seem to increase their expression of nestin upon activation (30). One potential problem in the identification of PaSCs is that the markers used can also be expressed by other cell types, including PaSC precursors, (myo)fibroblasts, vascular cells, and neural cells. However, on the basis of these markers,
PaSCs and HSCs are estimated to constitute nearly $4 \%$ and $8 \%$ of total pancreatic and hepatic cells, respectively $(3,21)$.

Although much has been learned from cultures of primary PaSCs, one of the dilemmas in the study of these cells is whether the transformation observed in culture is equivalent to what happens in pancreatic tissue (31). In culture, primary PaSCs continually change from a quiescent to an activated phenotype, and during this change they pass through a series of temporal states of transformation (32). For example, rapidly proliferating PaSCs in culture can either die by apoptosis or acquire a (myo)fibroblastic differentiated state that is more resistant to apoptosis. But how these observations relate to PaSC activation in vivo is unclear, which emphasizes the need for more information about the phenotypic states of PaSCs during disease progression and the mechanisms underlying the conversions between these states.

The propagation of immortalized PaSCs from rat and human pancreata provides additional experimental models to study PaSC biology (33-35) and provides a tool for overexpression and RNA interference studies, as well as a tool for high-throughput screening for compounds that affect PaSC activation. Immortalized cell lines have been generated by expression of either SV40 large $\mathrm{T}$ antigen alone in rat PaSCs or SV40 large T antigen and human telomerase in human PaSCs. The resultant immortalized cell lines possess a phenotype consistent with activated PaSCs, which includes expression of $\alpha$-SMA and ECM proteins. DNA microarrays have been used to compare the gene expression profile of immortalized and primary cultures of rat PaSCs. These revealed only a few overall differences, including differences in the expression of genes encoding ECM-related proteins, cytokines, integrins, and intermediate filament proteins (34). In addition, both rat and human immortalized cell lines responded to TGF- $\beta_{1}$, 
Table 1

General features of quiescent (inactive) and activated PaSCs

\begin{tabular}{|c|c|c|}
\hline & Quiescent & Activated \\
\hline \multicolumn{3}{|l|}{ Morphologic features } \\
\hline Vitamin A autofluorescence & + (in fat droplets) & Absent or remnant \\
\hline Endoplasmic reticulum & + & ++ \\
\hline Nucleus & Basal size & Enlarged \\
\hline \multicolumn{3}{|l|}{ Molecular markers ${ }^{A}$} \\
\hline Vimentin & t+ & +t \\
\hline Desmin & + & + \\
\hline GFAP & + & + \\
\hline Nestin & + & ++ \\
\hline$\alpha$-SMA & - & + \\
\hline \multicolumn{3}{|l|}{ Properties } \\
\hline ECM production & Limited & Prominent \\
\hline Cell proliferation & Limited & Increased \\
\hline Cell migration & Limited & Increased \\
\hline \multicolumn{3}{|c|}{$\begin{array}{l}- \text {, absence; }+ \text {, presence; }++, \text { present at high levels. AUpon isolation of } \\
\text { PaSCs, there is heterogeneity of marker expression that might reflect } \\
\text { multiple states of activation or differentiation, or different pools of fibro-- } \\
\text { genic cell type. Although } 100 \% \text { of cultured PaSCs isolated from rat } \\
\text { pancreas express vimentin, only } 20 \%-40 \% \text { of them express desmin } \\
\text { (4). The use of PaSC markers can suffer from similar limitations noted } \\
\text { for HSC markers. For example, } 70 \%-80 \% \text { of rat HSCs express des- } \\
\text { min, whereas human HSCs seem to lack desmin expression (21). }\end{array}$} \\
\hline
\end{tabular}

PDGF, and the PPAR $\gamma$ ligand $\mathrm{PGJ}_{2}$ in a manner similar to that of their cultured primary cell counterparts (34-36). The combined use of cultured primary PaSCs and immortalized cells, coupled with the use of coculture systems (for example, coculture of acinar cells and PaSCs), is likely to provide additional mechanistic insights into the biology of PaSCs.

\section{Mediators of PaSC activation}

The critical regulatory events that orchestrate the persistent activation of PaSCs in vivo are likely to be similar, at least in part, to the events that regulate the activation of primary PaSCs in culture. Studies of human and rodent primary PaSCs in culture have identified numerous growth factors, cytokines, hormones, intracellular signaling molecules, and transcription factors as regulators of PaSC activation. Potential activators of PaSCs in vivo include paracrine factors, such as cytokines (IL-1, IL-6, IL-8, and TNF- $\alpha$ ), growth factors (PDGF and TGF- $\beta_{1}$ ), angiotensin II, and reactive oxygen species released by damaged neighboring cells and leukocytes recruited in response to pancreatic injury (37-43) (Figure 3). Activated PaSCs, in turn, can produce autocrine factors, such as PDGF, TGF- $\beta_{1}$, cytokines (IL-1, IL-6, and TRAIL), and

\section{Figure 2}

Immune staining of PaSCs. (A and B) Normal mouse pancreas was triple stained to visualize GFAP (red), nuclei (blue), and keratin polypeptide 8 (green). L, lumen; arrows point to PaSC nuclei and arrowheads point to PaSC processes. (C-E) Pancreata from plasminogen-deficient mice injected with saline $(\mathbf{C})$ or with cerulein to induce pancreatitis ( $\mathbf{D}$ and $\mathbf{E}$ ) were stained with antibodies specific for $\alpha$-SMA as described previously (61). The image shown in $\mathbf{E}$ is a higher magnification of that shown in $\mathbf{D}$. Note the dramatic induction of $\alpha$-SMA in activated PaSCs that surround or are located between acini. Arrows point to blood vessel (v) staining. Scale bars: $20 \mu \mathrm{m}$ (A and B), $50 \mu \mathrm{m}$ (C and D), and $20 \mu \mathrm{m}$ (E). proinflammatory molecules (COX-2), that can perpetuate the activated phenotype $(40,42,44,45)$. In addition, activin-A, a member of the TGF- $\beta$ family of soluble factors, also functions in an autocrine manner, increasing collagen secretion and augmenting TGF- $\beta_{1}$ expression and secretion (46). Also, endothelin-1 is expressed by rat PaSCs in primary culture and can stimulate their migration and contraction (47). Although several inflammatory mediators released during pancreatitis have the potential to regulate PaSCs, abundant experimental evidence supports major roles for PDGF (which induces the proliferation of PaSCs and contributes to the migration potential of PaSCs) and TGF- $\beta_{1}$ and angiotensin II (which induce PaSCs to express $\alpha$-SMA and ECM proteins) as modulators of the persistently activated and profibrotic phenotype of these cells $(16-18,37,41)$ (Figure 3$)$.

Oxidative stress and ethanol metabolites have also been suggested as potential perpetuators of the activated PaSC phenotype. Ethanol can be metabolized in pancreatic acinar cells, resulting in toxic metabolites and oxidative stress that can induce pancreatic damage (48). Cultured rat PaSCs exhibit ethanol-induced alcohol dehydrogenase activity in vitro, suggesting that PaSCs can also metabolize ethanol (48). In vitro studies indicate that ethanol and its metabolite acetaldehyde promote the activation of rat PaSCs and cause lipid peroxidation in these cells $(48,49)$. Moreover, the antioxidant vitamin E prevents ethanol- and acetaldehydeinduced activation of PaSCs, thereby suggesting that oxidative stress regulates $\mathrm{PaSC}$ activation. For example, 4-hydroxy-nonenal, a highly reactive product of lipid peroxidation, activates primary rat PaSCs in culture. These in vitro findings were supported by histological analysis of pancreatic sections from patients with chronic pancreatitis, which showed 4-hydroxy-nonenal staining localized to activated PaSCs within fibrotic areas and to acinar cells adjacent to areas of fibrosis (28).

Multiple studies have identified several major signaling pathways involved in the regulation of PaSC function (27, 43, 47, 50-52). MAPKs are key mediators of activating signals initiated by growth factors, angiotensin II, and ethanol $(51,52)$. Other signaling pathways regulating PaSC activation include PI3K, RHO kinase, the JAK/STAT pathways, the activator protein-1 and NF- $\mathrm{B}$ pathways, and the TGF- $\beta /$ SMAD-related pathways (47,

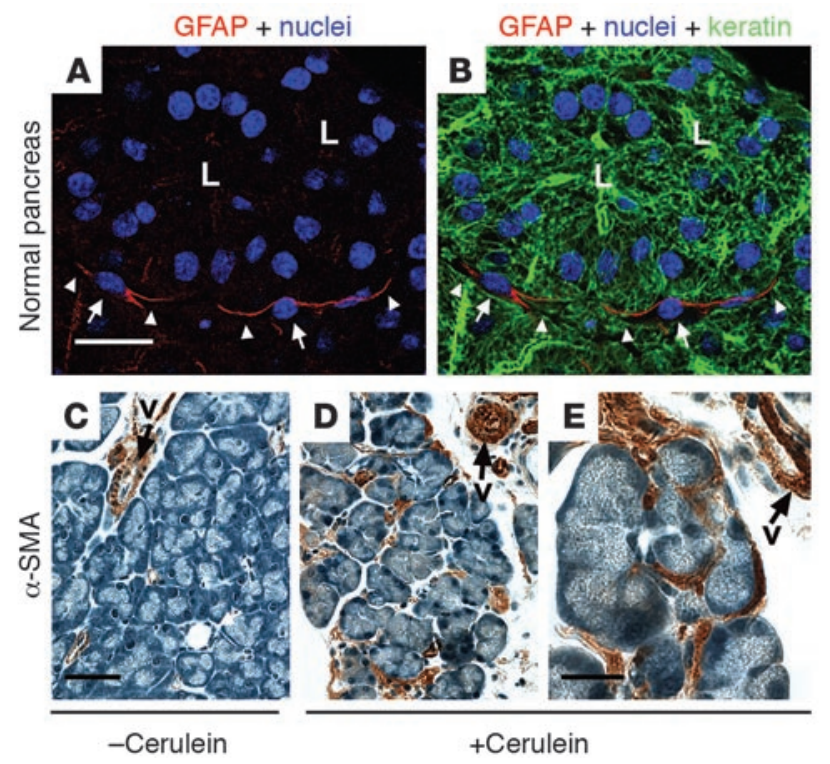




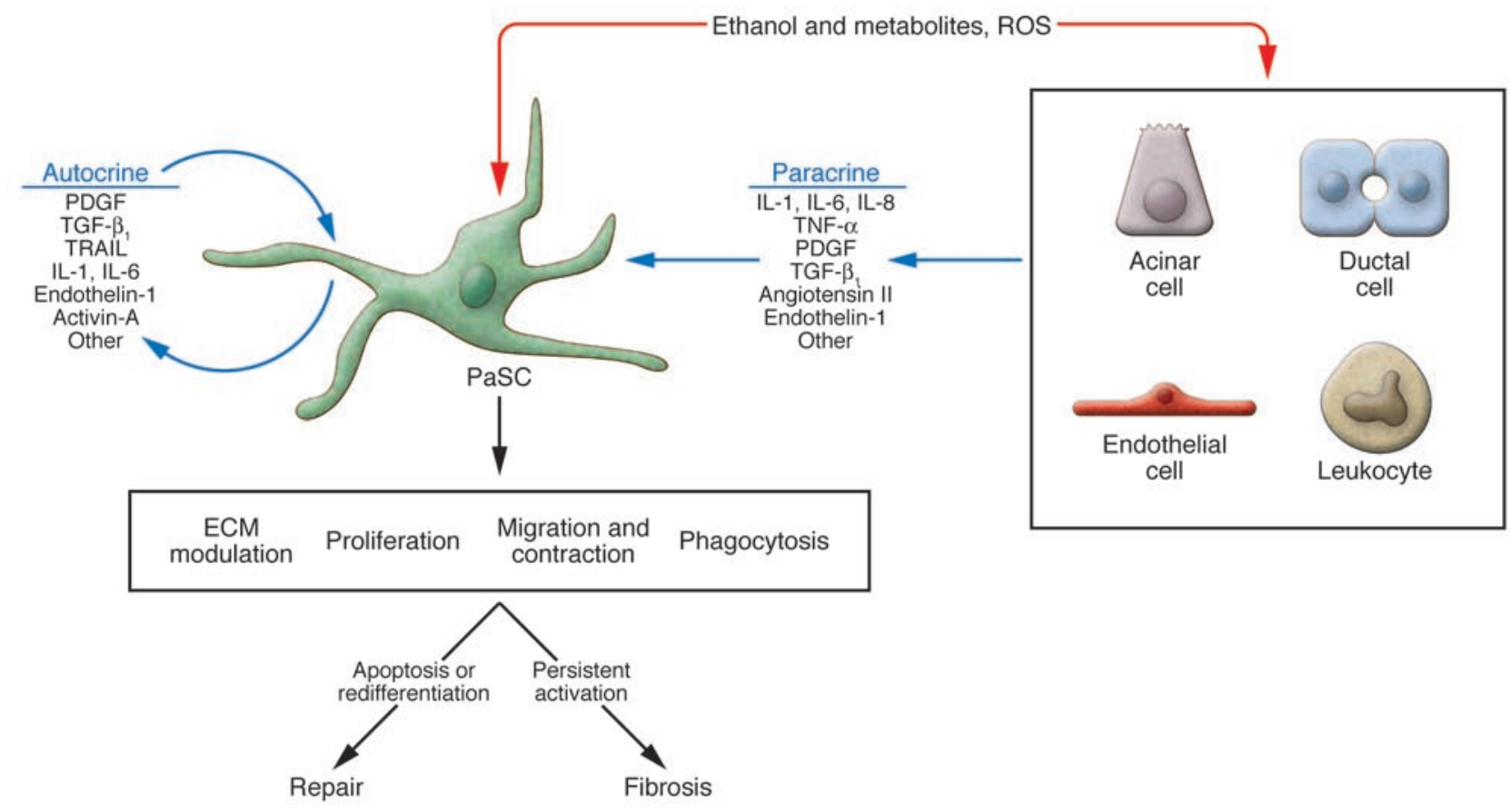

Figure 3

Mechanisms of PaSC activation. Exposure of the pancreas to ethanol, to its metabolites, and to insults that generate ROS all result in PaSC activation by autocrine and paracrine products. The paracrine factors are derived from neighboring cells such as acinar cells, ductal cells, endothelial cells, and leukocytes. Activated PaSCs can migrate to sites of tissue damage, undergo regulated contraction, proliferate, phagocytose, and generate products that modulate the ECM by facilitating repair or promoting fibrosis $(17,42,56,69)$. Persistent activation of PaSCs promotes fibrosis, while redifferentiation to a quiescent state or stimulation to undergo apoptosis facilitates tissue repair.

$50,53)$. In addition, studies using PPAR $\gamma$ ligands have implicated this pathway in the downregulation of PaSC activation (27). These signaling pathways are potential therapeutic targets for the modulation of PaSC function.

\section{PaSCs and pancreatic inflammation}

Extensive studies have shown that activated PaSCs in culture express several growth factors and cytokines, as well as the receptors for these molecules, which are known to participate in inflammatory and fibrotic processes. Although the cellular and molecular mechanisms underlying the development of pancreatitis are not completely understood, it is generally accepted that pancreatitis is initiated by damage to acinar, ductal, and/or mesenchymal cells in the pancreas $(7,54,55)$. Following damage, pathologic processes that occur in the pancreas include interstitial edema, necrosis of parenchymal cells, intrapancreatic trypsin activation, inflammatory cell infiltration, and activation and proliferation of PaSCs. In human and rodent pancreatic tissues, activated PaSCs are usually found in areas of extensive necrosis and inflammation where an environment rich in cytokines, growth factors, and reactive oxygen species prevails (56-58). Time-course studies using several animal models of experimental pancreatitis (59-61) indicate that parenchymal necrosis and inflammation precede PaSC activation, thereby suggesting that a necrotic, inflammatory process is a prerequisite for activation of these cells. Hence, autocrine and paracrine mediators are probably involved in PaSC activation. In turn, activation facilitates PaSC proliferation, migration, and ECM deposition, which leads to fibrosis or ECM remodeling as part of a repair process (Figure 3).
Evidence of a pivotal role for activated PaSCs in the development of pancreatic fibrosis is based, in part, on the analysis of pancreatic sections from patients with chronic pancreatitis and from experimental pancreatic fibrosis in rodents. For example, $\alpha$-SMA-expressing cells are abundant in areas of fibrosis in pancreatic tissue sections from patients with chronic pancreatitis of different etiologies $(4,27,28,58)$. In these fibrotic areas, only $\alpha$-SMA-expressing cells produce mRNA encoding procollagen $\alpha_{1} \mathrm{I}$, indicating that activated PaSCs are probably the predominant source of collagens during pancreatic fibrosis (27). Further evidence for this conclusion comes from the observation that spontaneous chronic pancreatitis in the Wistar Bonn/Kobori (Wbn/Kob) rat is characterized by inflammation and PaSC activation in areas of pancreatic fibrosis (50). In addition, in rats, administration of trinitrobenzene sulfonic acid into the pancreatic duct, as well as i.v. injection of dibutyltin dichloride, induces necrosis and inflammation of the pancreas followed by PaSC activation and fibrosis (27, 62). Similarly, mouse pancreatitis induced with the use of cerulein (a cholecystokinin analogue and pancreas secretagogue) is also accompanied by PaSC activation and fibrosis $(63,64)$.

The involvement of PaSCs in the regulation of the inflammatory response during pancreatitis occurs, at least in part, through their production of chemokines and cytokines, as has been reported for myofibroblast-type cells in other tissues (65-68). PaSCs have also been shown to have phagocytic activity in vivo and in cell culture (69) and therefore might also function as resident phagocytic cells during pancreatitis. PaSC phagocytic activity is regulated by PPAR $\gamma$ and expression of CD36, a scavenger receptor that promotes phagocytosis. Interestingly, profibrogenic cytokines, such 
as TGF- $\beta$, TNF- $\alpha$, and IL-1, decreased the phagocytic capacity of PaSCs activated in culture (69). These results highlight the complex regulation of the profibrotic and proinflammatory properties of PaSCs during pancreatic inflammation.

\section{Inflammation and PaSC modulation of the ECM}

PaSCs have a key role in the extensive tissue fibrosis that accompanies chronic pancreatitis and leads to destruction of the pancreas and loss of exocrine function $(17,56,58)$. Conversely, observational studies have shown that activated PaSCs participate in tissue repair processes after acute necrotizing pancreatitis in humans and experimental acute pancreatitis in rodents (59-61, 70). Although the exact role of PaSCs in pancreatic repair and regeneration remains to be elucidated, activated PaSCs and other myofibroblast cells resident in the pancreas probably contribute to the formation of a provisional matrix at the site of injury that allows cell proliferation, migration, and assembly of new parenchymal cells $(60,61,71)$. In addition, PaSCs can regulate ECM remodeling during pancreatic tissue repair through the production of ECMdegrading proteases and their inhibitors, such as tissue inhibitor of metalloproteinase 1 (TIMP-1) $(40,72)$. In this context, resolution of mouse cerulein-induced pancreatitis involves transient activation of PaSCs and deposition of ECM proteins, as well as transient upregulation of MMPs and TIMP-1 (61). Similar regulation of ECM remodeling by HSCs is also likely to mediate liver regeneration after experimental injury $(20,73)$.

In most studies in which PaSCs are activated after damage to the pancreas, the inflammatory process resolves and activated PaSCs progressively disappear after the cessation of the injurious agent. However, repeated pancreatic damage and failure of the mechanisms regulating tissue repair can both lead to chronic inflammation, persistent activation, proliferation of PaSCs, and finally fibrosis. Consistent with this hypothesis, repeated episodes of acute experimental pancreatitis produce changes that resemble those found in chronic pancreatitis $(63,74)$. In fact, fibrosis in the pancreas and other organs can be considered the consequence of a wound-healing process responding to chronic injurious stimuli. In humans, repeated damage to the pancreas is associated with chronic alcohol consumption, pancreatic duct obstruction, metabolic disorders, and genetic defects $(7,75)$. The chronic injury results in perpetuation of the activated PaSC phenotype. Furthermore, chronic pancreatitis is associated with reduced production of MMPs by PaSCs, which probably helps promote and sustain the fibrotic phenotype (40).

In addition to the multiple factors described above, other mechanisms can explain the persistent activated state of PaSCs during pancreatitis. For example, activated PaSCs express proteaseactivated receptor-2 (PAR-2), which is cleaved by trypsin (a key pathogenic protease in pancreatitis) to become active. Active PAR-2 stimulates PaSC proliferation and collagen synthesis (76). Changes in the composition of the ECM during repair processes can also modulate PaSC activation. For example, activated PaSCs revert to a quiescent state when cultured on a basal membranelike matrix, thereby suggesting that ECM composition regulates PaSC behavior $(35,40)$. Studies of liver fibrosis have shown that extensive ECM degradation is accompanied by apoptosis of HSCs, as a result of either increased proapoptotic signaling or reduced survival signals from the ECM (77), but it remains to be shown whether this is also true in the pancreas. More recently, impaired extracellular proteolysis of the ECM in mice lacking plasminogen has been associated with persistent PaSC activation and accumulation of pancreatic collagens during the recovery phase of mouse cerulein-induced pancreatitis (61). Moreover, levels of pancreatic TGF- $\beta_{1}$, plasminogen activator inhibitor- 1 , and TIMP-1, factors that promote fibrosis $(37,77)$, were elevated persistently in plasminogen-deficient mice after treatment with cerulein but only transiently in similarly treated plasminogen-sufficient mice (61).

\section{PaSCs and pancreatic cancer}

Ductal pancreatic adenocarcinoma, which is the most common form of pancreatic cancer, is the fourth leading cause of cancerrelated death, with a 5-year survival of less than 5\% (78). Several tumors, and in particular pancreatic adenocarcinomas, are characterized by "tumor desmoplasia," a remarkable increase in connective tissue that infiltrates and envelopes the neoplasm (79). Activated PaSCs in the tumor desmoplasia of human pancreatic cancers express $\alpha$-SMA and colocalize with mRNA encoding procollagen $\alpha_{1} \mathrm{I}$ (14) and are probably major contributors of the ECM proteins that constitute the desmoplasia $(12,14,80-84)$. Of note, analysis of gene expression in human pancreatic adenocarcinoma, chronic pancreatitis, normal pancreas, and pancreatic cancer cell lines demonstrated that 107 genes predicted to be expressed in the stromal compartment were found in both pancreatic adenocarcinoma and chronic pancreatitis (81). Furthermore, isolation and characterization of stromal cells from human pancreatic adenocarcinoma and alcohol-induced chronic pancreatitis samples demonstrated that cells from both sources had the same characteristic morphology, cytofilament expression, and capacity to synthesize ECM proteins (84). These results demonstrate that these 2 disorders contain common stromal elements and suggest similar mechanisms underlying the development of fibrosis in chronic pancreatitis and the desmoplasia in pancreatic adenocarcinoma.

Evidence is emerging that there is a symbiotic relationship between pancreatic adenocarcinoma cells and PaSCs that results in an overall increase in the rate of growth of the tumor (Figure 4). For example, culture supernatants from human pancreatic tumor cell lines stimulate PaSC proliferation and production of ECM proteins $(14,82-84)$. In addition, the growth rate of tumor cells injected s.c. into nude mice (mice that lack $\mathrm{T}$ cells and are severely immunocompromised) is markedly increased when PaSCs are included in the inoculum (84). In contrast to the tumors that form when only cancer cells are injected, the tumors that form when both cancer cells and PaSCs are used have a desmoplasia similar to that observed in human pancreatic adenocarcinoma (84). Furthermore, pancreatic tumor cells induce the proliferation of PaSCs by secreting PDGF and induce PaSC production of ECM proteins by secreting TGF- $\beta_{1}$ and FGF-2 (84).

Although studies using animals indicate that pancreatic tumor cells and PaSCs promote each other's proliferation, supporting data from human pancreatic tumors are limited and inconsistent. One report shows that more extensive intratumoral fibroblastic cell proliferation correlates with a poorer disease outcome (85). Another report shows that patients with a better outcome have increased expression of connective tissue growth factor (CTGF) in fibroblasts surrounding the human pancreatic tumor cells (86). CTGF has been implicated in the pathogenesis of fibrotic diseases, and its expression is predominant in PaSCs and is regulated by TGF- $\beta$. These early findings are intriguing and suggest that further work is needed to delineate the role of PaSCs and their regulators in pancreatic cancer. 


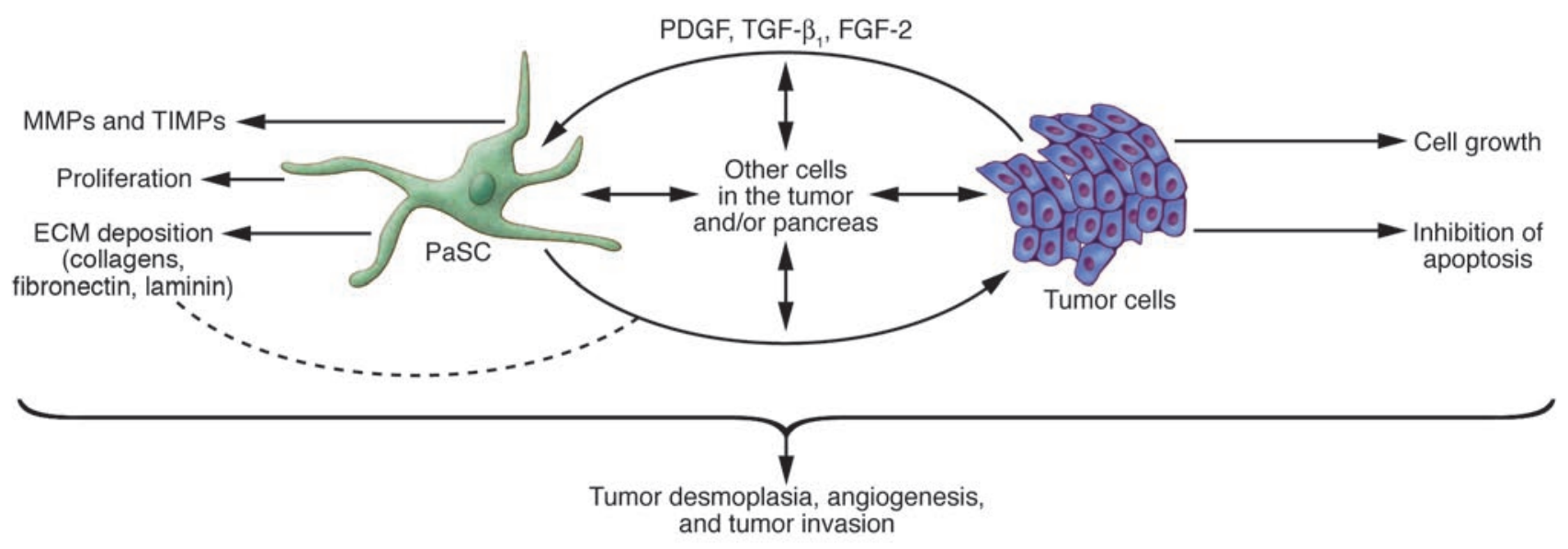

Figure 4

Effect of PaSCs on tumor cell invasion and desmoplasia. There is accumulating evidence for PaSC cross-talk with tumor cells, which in turn contributes to the profound tumor desmoplasia that is noted in pancreatic cancer (12, 14, 80-84). Such direct or indirect cell-cell dialogue can also promote tumor invasion and possibly angiogenesis, although a role in angiogenesis has been more studied in the liver. Other cells in the pancreas (for example acinar cells, ductal cells, endothelial cells, and leukocytes) are likely to be involved.

The mechanisms by which PaSCs and the desmoplasia enhance the growth of tumor cells in the adenocarcinomas are complex and only partly understood. One role of the desmoplasia is to promote survival and prevent apoptosis of the tumor cells through a direct action of ECM proteins on the tumor cells $(87,88)$ (Figure 4). The prosurvival effects of the ECM proteins laminin and fibronectin are mediated through their integrin receptors, which are expressed by the tumor cells. In addition, the effects of fibronectin seem to be mediated through transactivation of IGF-1. Overall, these interactions lead to the activation of prosurvival and progrowth signaling pathways in pancreatic tumor cells $(88,89)$.

Another possible mechanism by which tumor desmoplasia might promote pancreatic adenocarcinoma cell growth is that PaSCs and tumor cells produce MMPs and tissue serine proteases (Figure 4), such as members of the plasminogen activator system, that degrade ECM proteins $(61,72,90)$. In this context, MMPs and tissue serine proteases might promote tumor cell invasion and metastasis, as has been postulated in other cancers (90-93).

\section{Comparison of PaSCs and HSCs}

Although PaSCs and HSCs share several morphologic and marker features, some differences exist. Unique, context-related features of HSCs include the portal perfusion and generally abundant vascular flow in the liver, and their proximity to encounter endothelial cell cross-talk due to their hepatic location within the subendothelial space (19). In addition, a genome-wide assessment of gene expression that included 21,329 genes identified 29 that were differentially expressed between cultures of primary HSCs and PaSCs (94). However, the fact that the overwhelming majority of genes showed no difference in expression supports the notion that these 2 cell types are very similar. Recent studies have revealed that the bone marrow is the source of $68 \%$ of HSCs and $70 \%$ of myofibroblast cells in mouse models of carbon tetrachloride- and thioacetamide-induced fibrosis (95). Whether the same holds true in the pancreas remains to be determined, but a common origin in the bone marrow could account for the strong similarities between HSCs and PaSCs.

Although this Review has concentrated on stellate cells, other potential sources of fibrosis are present in both the pancreas and the liver. For example, hepatic myofibroblasts, which are distinguished from HSCs by their location within the liver and their expression of the ECM component fibulin-2 but not GFAP (96, 97), and tissue fibroblasts, which express vimentin but not GFAP and desmin, represent an additional source of ECM proteins (96-100). Subpopulations of fibroblastic cells, which might reflect distinct levels of fibroblast activation, can be distinguished in cirrhotic livers on the basis of differing levels of cellular retinol-binding protein- 1 and $\alpha$-SMA (101), and these cells might also produce ECM proteins. The origin of these liver myofibroblast-type cells and similar cells in the pancreas remains to be conclusively determined. Bone marrow transplantation in a mouse model of pancreatic insulinoma demonstrated that the bone marrow is the source of $25 \%$ of pancreatic myofibroblasts and is likely to contribute to tumor-associated fibrosis (102). In addition, nestin-positive and nestin-negative precursors isolated from adult mouse pancreas are able to differentiate in vitro to yield multiple lineages of the pancreas, including PaSCs (103). The relative contributions of bone marrow and resident precursors to the PaSC population, as well as to the other myofibroblast- and fibroblast-related cell populations, remain to be determined.

The function of quiescent PaSCs is still being investigated, but clues can be gleaned from studies involving HSCs (20-22). For example, HSCs have been implicated in several important liver physiologic roles, under basal conditions, that include vitamin A storage; production and turnover of normal ECM proteins; and communication with hepatocytes via gap junctions and the production of paracrine factors to promote hepatocyte differentiation, and to regulate ductal and vascular pressures. Similar roles can be envisioned for PaSCs in the inactive state. For example, perivascular and periductal PaSCs (2) might regulate the pressure within these compartments by contraction mediated by endothelin-1 (47). The functions of PaSCs, by analogy to HSCs, should be envisioned not only in isolation but also in the context and under the influence of their microenvironment.

Although alcohol and/or its metabolites have been shown to similarly activate stellate cells of the pancreas and liver, it is unclear why substantial differences exist in an individual's sus- 


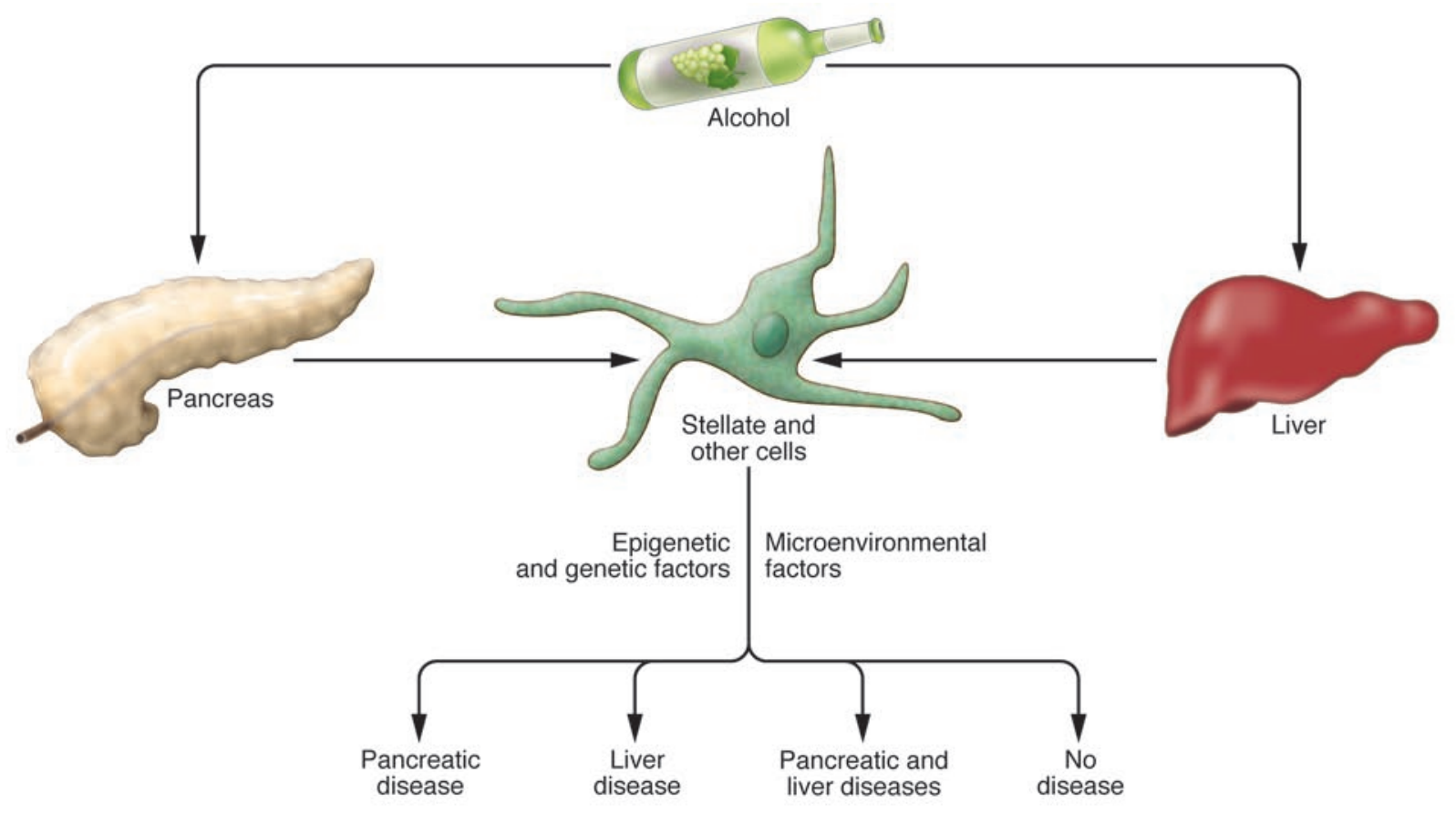

Figure 5

Differential impact of alcohol on the pancreas and liver. The effects of alcohol and its metabolites on the pancreas and liver can vary substantially from one individual to another, with some showing no effects, some having a pancreas-selective or liver-selective effect, and yet others having disease in both organs. Poorly understood genetic, epigenetic, and microenvironmental factors are likely to be responsible for these dramatic differences.

ceptibility to liver and pancreas fibrosis (Figure 5). Potential differences between the pancreas and the liver after injury, in addition to the apparent difference in the cellular microenvironment, include edema formation during pancreatitis; different ethanol metabolism in acinar cells and hepatocytes; and different lipid metabolism between acinar cells and hepatocytes (for example, "fatty" pancreas has not been described). Given that the stellate cell is an integral element of the onset of these diseases, microenvironment differences and other genetic and epigenetic modifiers related to stellate and/or other resident and infiltrating cells are likely to be important (Figure 5), but this hypothesis remains to be tested. For example, comparison of the response of PaSCs and HSCs isolated from the same animal with parallel comparative responses in different genetic backgrounds has not been reported. Furthermore, attempts at assessing genetic polymorphisms (for example, in enzymes such as cytochrome P450 2E1 and aldehyde dehydrogenase-2) that may promote susceptibility to alcoholic chronic pancreatitis or cirrhosis have not been overly revealing (104). However, an understanding of genetic polymorphisms that predispose to chronic pancreatitis $(8,75)$ or liver fibrosis $(105$, $106)$, or even protect from chronic pancreatitis (107), is being pursued. A systematic and broad-based genetic screening will be needed, using well-defined animal models and patient cohorts. Proteomic comparisons between PaSCs and HSCs, which are likely to be forthcoming, should also provide additional insights.

\section{Potential treatments for PaSC disorders}

In concept, treatments for disorders involving PaSCs (that is, chronic pancreatitis and pancreatic cancer) should target the key mechanisms involved in their activation and proliferation.
For example, blockade of the receptors for PDGF, TGF- $\beta$, and angiotensin II, as well as blockade of the intracellular signaling pathways downstream of these receptors, is likely to provide therapeutic benefit $(17,51,76,108)$. In this regard, there are in vivo experiments showing the importance of the angiotensin II system in the development of pancreatic fibrosis $(64,108)$. There are also several reports from in vitro experiments using PaSCs that show key roles in the activation and/or proliferation process for MAPK pathways, in particular, ERK1/2, p38 kinase, and JNK (50, 52, 53, 66, 109, 110); PI3K and PKC (111); PPAR $\gamma(36,112)$; NADPH oxidases (our unpublished observations); and ethanol metabolism to acetaldehyde (18). In these studies, inhibition of most of these pathways results in attenuation of the activation and proliferation of PaSCs, but activation of PPAR $\gamma$ seems to block PaSC activation $(36,112)$. It has also been reported that activated rat PaSCs express COX- 2 when stimulated with TGF- $\beta_{1}$ and other cytokines (45), as well as when stimulated with conditioned media from human pancreatic tumor cells (113). Pharmacological inhibition of COX- 2 and blockade of the TGF- $\beta_{1}$ signaling pathway decrease the expression of COX-2, $\alpha$-SMA, and collagen I, suggesting that COX-2 might be a relevant therapeutic target for chronic pancreatitis and pancreatic cancer. Furthermore, efforts aimed at inducing PaSC transdifferentiation from an activated to a quiescent state and at inducing PaSC apoptosis are also attractive modalities. For example, administration of vitamin A (retinol and its metabolites) induces culture-activated rat PaSCs to become quiescent (114).

In vivo studies using animal models of chronic pancreatitis have demonstrated that several agents have beneficial effects (115), but few in vivo studies have assessed whether targeting PaSCs in the 
desmoplastic reaction of pancreatic cancer has beneficial effects. In the case of targeting fibrosis in pancreatic tumor models, antibodies to CTGF, which activate PaSCs through their $\alpha_{5} \beta_{1}$ integrin receptor (44), inhibited tumor growth and metastasis in a xenograft mouse pancreatic tumor model $(116,117)$. Agents showing benefit in experimental chronic pancreatitis include inhibitors of angiotensin II-converting enzyme and antagonists of the angiotensin II receptor (50, 108); vitamin E (118) and DA-9601 (119), which function as antioxidants; troglitazone and thiazolidinedione, which are PPAR $\gamma$ ligands $(120,121)$; camostat, which is a serine protease inhibitor $(62,122)$; the herbal preparation Saiko-keishito, which has antioxidant and antiinflammatory properties (123); and COX-2 inhibitors (124). In addition, strategies aimed at blocking TGF- $\beta$ signaling pathways $(113,125)$ have proved effective in reducing experimental pancreatic fibrosis in rodents. Considering the importance of PaSC pathobiology in both chronic pancreatitis and pancreatic cancer, there should be increased effort devoted to addressing PaSC functions in these disorders and the development of therapeutic strategies that include targeting the PaSC.

\section{Concluding remarks}

The PaSC is a recently identified cell in the pancreas, which explains why a PubMed search for "pancreatic stellate cells" provides 177 total citations, with a substantially rising trajectory (for example, 64 citations in 2004 and 2005 versus 45 citations in 2003 and 2004), as compared with 1,806 total citations for "hepatic stellate cells." Presently, much remains to be learned about the biology of PaSCs and their role in diseases of the pancreas. Indeed, we need a better understanding of their function during the qui- escent state, their regulation in terms of activation and deactivation (that is, differentiation and transdifferentiation, respectively), their elimination (for example, through apoptosis), their cross-talk with neighboring cells, and their origin. Such understanding will promote and refine therapeutic approaches targeting PaSCs for disorders such as pancreatitis and pancreatic cancers.

\section{Acknowledgments}

We are very grateful to Diana Toivola for preparing the immunofluorescence figure and for input during manuscript preparation; Yoon Jung and Carolyn Taylor for assistance with the references; Kris Morrow for preparing the figures; and Minote Apte, Scott Friedman, Roland Schmid, and Hide Tsukamoto for essential comments on the manuscript. Our work is supported by NIH grants DK52951, DK47918, and DK73909 and a VA Merit Award (to M.B. Omary); Stanford University NIH Digestive Disease Center grant DK56339; NIH grant AA15781 (to A. Lugea); the Susan E. Riley and Edna E. Riley Charitable Foundation (to A.W. Lowe); and USCUCLA NIH Research Center for Alcoholic Liver and Pancreatic Diseases award AA11999. We apologize for being unable to include all the references related to this field because of space limitation.

Address correspondence to: M. Bishr Omary, Department of Medicine, VA Palo Alto Health Care System, 3801 Miranda Avenue, 154J, Palo Alto, California 94304, USA. Fax: (650) 852-3259; E-mail: mbishr@stanford.edu. Or to: Stephen J. Pandol, Veterans Affairs Greater Los Angeles Healthcare System, 11301 Wilshire Boulevard, Building 258, Room 340, Los Angeles, California 90073, USA. Fax: (310) 268-4578; E-mail: stephen.pandol@med.va.gov.
1. Watari, N., Hotta, Y., and Mabuchi, Y. 1982. Morphological studies on a vitamin A-storing cell and its complex with macrophage observed in mouse pancreatic tissues following excess vitamin A administration. Okajimas Folia Anat. Jpn. 58:837-858.

2. Ikejiri, N. 1990. The vitamin A-storing cells in the human and rat pancreas. Kurume Med. J. 37:67-81.

3. Apte, M.V., et al. 1998. Periacinar stellate shaped cells in rat pancreas: identification, isolation, and culture. Cancer Res. 43:128-133.

4. Bachem, M.G., et al. 1998. Identification, culture, and characterization of pancreatic stellate cells in rats and humans. Gastroenterology. 115:421-432.

5. Whitcomb, D.C. 2004. Inflammation and cancer. V. Chronic pancreatitis and pancreatic cancer. Am.J. Physiol. Gastrointest. Liver Physiol. 287:G315-G319.

6. Draganov, P., and Forsmark, C.E. 2005. "Idiopathic" pancreatitis. Gastroenterology. 128:756-763.

7. Dimagno, M.J., and Dimagno, E.P. 2006. Chronic pancreatitis. Curr. Opin. Gastroenterol. 22:487-497.

8. Weiss, F.U., Simon, P., Mayerle, J., Kraft, M., and Lerch, M.M. 2006. Germline mutations and gene polymorphism associated with human pancreatitis. Endocrinol. Metab. Clin. North Am. 35:289-302, viii-ix.

9. Comfort, M.W., Gambill, E.E., and Baggenstoss, A.H. 1946. Chronic relapsing pancreatitis: a study of 29 cases without associated disease of the biliary or gastro-intestinal tract. Gastroenterology. 6:239-285.

10. Lowenfels, A.B., et al. 1993. Pancreatitis and the risk of pancreatic cancer. International Pancreatitis Study Group. N. Engl. J. Med. 328:1433-1437.

11. Go, V.L., Gukovskaya, A., and Pandol, S.J. 2005. Alcohol and pancreatic cancer. Alcohol. 35:205-211.

12. Yen, T.W., et al. 2002. Myofibroblasts are responsible for the desmoplastic reaction surrounding human pancreatic carcinomas. Surgery. 131:129-134.

13. Logsdon, C.D., et al. 2003. Molecular profiling of pancreatic adenocarcinoma and chronic pancreatitis identifies multiple genes differentially regulated in pancreatic cancer. Cancer Res. 63:2649-2657.

14. Apte, M.V., et al. 2004. Desmoplastic reaction in pancreatic cancer: role of pancreatic stellate cells. Pancreas. 29:179-187.

15. Schneider, G., Siveke, J.T., Eckel, F., and Schmid, R.M. 2005. Pancreatic cancer: basic and clinical aspects. Gastroenterology. 128:1606-1625

16. Menke, A., and Adler, G. 2002. TGFbeta-induced fibrogenesis of the pancreas. Int.J. Gastrointest. Cancer. 31:41-46.

17. Jaster, R. 2004. Molecular regulation of pancreatic stellate cell function. Mol. Cancer. 3:26.

18. Apte, M.V., and Wilson, J.S. 2004. Mechanisms of pancreatic fibrosis. Dig. Dis. 22:273-279.

19. Friedman, S.L. 1993. Seminars in medicine of the Beth Israel Hospital, Boston. The cellular basis of hepatic fibrosis. Mechanisms and treatment strategies. N. Engl. J. Med. 328:1828-1835.

20. Bissell, D.M. 1998. Hepatic fibrosis as wound repair: a progress report. J. Gastroenterol. 33:295-302.

21. Geerts, A. 2001. History, heterogeneity, developmental biology, and functions of quiescent hepatic stellate cells. Semin. Liver Dis. 21:311-335.

22. Tsukamoto, H. 2005. Adipogenic phenotype of hepatic stellate cells. Alcohol. Clin. Exp. Res. 29(Suppl. 11):132S-133S

23. Liu, Y. 2006. Renal fibrosis: new insights into the pathogenesis and therapeutics. Kidney Int. 69:213-217.

24. Keane, M.P., Strieter, R.M., and Belperio, J.A. 2005. Mechanisms and mediators of pulmonary fibrosis. Crit. Rev. Immunol. 25:429-463.

25. Wake, K. 1980. Perisinusoidal stellate cells (fat-storing cells, interstitial cells, lipocytes), their related structure in and around the liver sinusoids, and vitamin A-storing cells in extrahepatic organs. Int. Rev. Cytol. 66:303-353.

26. Omary, M.B., Coulombe, P.A., and McLean, W.H 2004. Intermediate filament proteins and their associated diseases. N. Engl. J. Med. 351:2087-2100.

27. Haber, P.S., et al. 1999. Activation of pancreatic stellate cells in human and experimental pancreatic fibrosis. Am. J. Pathol. 155:1087-1095.

28. Casini, A., et al. 2000. Collagen type I synthesized by pancreatic periacinar stellate cells (PSC) co-localizes with lipid peroxidation-derived aldehydes in chronic alcoholic pancreatitis. J. Pathol. 192:81-89.

29. Saotome, T., Inoue, H., Fujimiya, M., Fujiyama, Y., and Bamba, T. 1997. Morphological and immunocytochemical identification of periacinar fibroblast-like cells derived from human pancreatic acini. Pancreas. 14:373-382.

30. Lardon, J., Rooman, I., and Bouwens, L. 2002. Nestin expression in pancreatic stellate cells and angiogenic endothelial cells. Histochem. Cell Biol. 117:535-540.

31. Pandol, S.J. 2005. Are we studying the correct state of the stellate cell to elucidate mechanisms of chronic pancreatitis? Gut. 54:744-745.

32. Manapov, F., Muller, P., and Rychly,J. 2005. Translocation of 2 21(Cip1/WAF1) from the nucleus to the cytoplasm correlates with pancreatic myofibroblast to fibroblast cell conversion. Gut. 54:814-822.

33. Masamune, A., Satoh, M., Kikuta, K., Suzuki, N., and Shimosegawa, T. 2003. Establishment and characterization of a rat pancreatic stellate cell line by spontaneous immortalization. World J. Gastroenterol. 9:2751-2758.

34. Sparmann, G., et al. 2004. Generation and characterization of immortalized rat pancreatic stellate cells. Am. J. Physiol. Gastrointest. Liver Physiol. 287:G211-G219.

35. Jesnowski, R., et al. 2005. Immortalization of pancreatic stellate cells as an in vitro model of pancreatic fibrosis: deactivation is induced by matrigel and $\mathrm{N}$-acetylcysteine. Lab. Invest. 85:1276-1291.

36. Jaster, R., et al. 2005. Peroxisome proliferator-activated receptor gamma overexpression inhibits pro- 
fibrogenic activities of immortalised rat pancreatic stellate cells. J. Cell. Mol. Med. 9:670-682.

37. Apte, M.V., et al. 1999. Pancreatic stellate cells are activated by proinflammatory cytokines: implications for pancreatic fibrogenesis. Gut. 44:534-541.

38. Luttenberger, T., et al. 2000. Platelet-derived growth factors stimulate proliferation and extracellular matrix synthesis of pancreatic stellate cells: implications in pathogenesis of pancreas fibrosis. Lab. Invest. 80:47-55.

39. Schneider, E., et al. 2001. Identification of mediators stimulating proliferation and matrix synthesis of rat pancreatic stellate cells. Am. J. Physiol. Cell Physiol. 281:C532-C543.

40. Shek, F.W., et al. 2002. Expression of transforming growth factor-beta 1 by pancreatic stellate cells and its implications for matrix secretion and turnover in chronic pancreatitis. Am. J. Pathol. 160:1787-1798.

41. Mews, P., et al. 2002. Pancreatic stellate cells respond to inflammatory cytokines: potential role in chronic pancreatitis. Gut. 50:535-541.

42. Phillips, P.A., et al. 2003. Cell migration: a novel aspect of pancreatic stellate cell biology. Gut. 52:677-682.

43. Hama, K., et al. 2006. Angiotensin II promotes the proliferation of activated pancreatic stellate cells by Smad7 induction through a protein kinase C pathway. Biochem. Biophys. Res. Commun. 340:742-750.

44. Gao, R., and Brigstock, D.R. 2005. Connective tissue growth factor (CCN2) in rat pancreatic stellate cell function: integrin alpha5beta 1 as a novel CCN2 receptor. Gastroenterology. 129:1019-1030.

45. Aoki, H., et al. 2007. Cyclooxygenase-2 is required for activated pancreatic stellate cells to respond to pro-inflammatory cytokines. Am. J. Physiol. Cell Physiol. In press.

46. Ohnishi, N., et al. 2003. Activin A is an autocrine activator of rat pancreatic stellate cells: potential therapeutic role of follistatin for pancreatic fibrosis. Gut. 52:1487-1493.

47. Masamune, A., Satoh, M., Kikuta, K., Suzuki, N., and Shimosegawa, T. 2005. Endothelin-1 stimulates contraction and migration of rat pancreatic stellate cells. World J. Gastroenterol. 11:6144-6151.

48. Apte, M.V., Pirola, R.C., and Wilson, J.S. 2006. Battle-scarred pancreas: role of alcohol and pancreatic stellate cells in pancreatic fibrosis. J. Gastroenterol. Hepatol. 21(Suppl. 3):S97-S101.

49. Pandol, S., et al. 2006. Alcohol, reactive oxygen species, pancreatitis and pancreatic cancer. In Alcohol, tobacco, and cancer. C.H. Cho and V. Purohit, editors. Karger. Basel, Switzerland. 109-118.

50. Kuno, A., et al. 2003. Angiotensin-converting enzyme inhibitor attenuates pancreatic inflammation and fibrosis in male Wistar Bonn/Kobori rats. Gastroenterology. 124:1010-1019.

51. Ohnishi, H., et al. 2004. Distinct roles of Smad2-, Smad3-, and ERK-dependent pathways in transforming growth factor-beta1 regulation of pancreatic stellate cellular functions. J. Biol. Chem. 279:8873-8878.

52. Kikuta, K., et al. 2006. Hydrogen peroxide activates activator protein-1 and mitogen-activated protein kinases in pancreatic stellate cells. Mol. Cell. Biochem. 29:111-120.

53. Masamune, A., et al. 2004. A c-Jun NH2-terminal kinase inhibitor SP600125 (anthra[1,9-cd]pyrazole-6 $(2 \mathrm{H})$-one) blocks activation of pancreatic stellate cells. J. Pharmacol. Exp. Ther. 310:520-527.

54. Stevens, T., Conwell, D.L., and Zuccaro, G. 2004. Pathogenesis of chronic pancreatitis: an evidencebased review of past theories and recent developments. Am. J. Gastroenterol. 99:2256-2270.

55. Ruthenburger, M., Mayerle, J., and Lerch, M.M. 2006. Cell biology of pancreatic proteases. Endocrinol. Metab. Clin. North Am. 35:313-331, ix.

56. Apte, M.V., and Wilson, J.S. 2003. Stellate cell activation in alcoholic pancreatitis. Pancreas. 27:316-320.
57. Bentrem, D.J., and Joehl, R.J. 2003. Pancreas: healing response in critical illness. Crit. Care Med. 31(Suppl. 8):S582-S589.

58. Kloppel, G., Detlefsen, S., and Feyerabend, B. 2004 Fibrosis of the pancreas: the initial tissue damage and the resulting pattern. Virchows Arch. 445:1-8.

59. Yokota, T., et al. 2002. Pancreatic stellate cell activation and MMP production in experimental pancreatic fibrosis. J. Surg. Res. 104:106-111.

60. Zimmermann, A., et al. 2002. Pancreatic stellate cells contribute to regeneration early after acute necrotising pancreatitis in humans. Gut. 51:574-578.

61. Lugea, A., et al. 2006. Pancreas recovery following cerulein-induced pancreatitis is impaired in plasminogen-deficient mice. Gastroenterology. 131:885-899.

62. Gibo, J., et al. 2005. Camostat mesilate attenuates pancreatic fibrosis via inhibition of monocytes and pancreatic stellate cells activity. Lab. Invest. 85:75-89.

63. Neuschwander-Tetri, B.A., Bridle, K.R., Wells, L.D., Marcu, M., and Ramm, G.A. 2000. Repetitive acute pancreatic injury in the mouse induces procollagen alpha1(I) expression colocalized to pancreatic stellate cells. Lab. Invest. 80:143-150.

64. Nagashio, Y., et al. 2004. Angiotensin II type 1 receptor interaction is an important regulator for the development of pancreatic fibrosis in mice. Am. J. Physiol. Gastrointest. Liver Physiol. 287:G170-G177.

65. Andoh, A., et al. 2000. Cytokine regulation of chemokine (IL-8, MCP-1, and RANTES) gene expression in human pancreatic periacinar myofibroblasts. Gastroenterology. 119:211-219.

66. Masamune, A., Kikuta, K., Satoh, M., Satoh, A., and Shimosegawa, T. 2002. Alcohol activates activator protein-1 and mitogen-activated protein kinases in rat pancreatic stellate cells. J. Pharmacol. Exp. Ther. 302:36-42.

67. Sparmann, G., et al. 2005. Inhibition of lymphocyte apoptosis by pancreatic stellate cells: impact of interleukin-15. Am. J. Physiol. Gastrointest. Liver Physiol. 289:G842-G851.

68. Aoki, H., et al. 2006. Autocrine loop between TGFbeta 1 and IL-1beta through Smad3- and ERKdependent pathways in rat pancreatic stellate cells. Am. J. Physiol. Cell Physiol. 290:C1100-C1108.

69. Shimizu, K., Kobayashi, M., Tahara, J., and Shiratori, K. 2005. Cytokines and peroxisome proliferator-activated receptor gamma ligand regulate phagocytosis by pancreatic stellate cells. Gastroenterology. 128:2105-2118.

70. Kishi, S., et al. 2003. Pancreatic duct obstruction itself induces expression of alpha smooth muscle actin in pancreatic stellate cells. J. Surg. Res. 114:6-14.

71. Demols, A., et al. 2002. Endogenous interleukin-10 modulates fibrosis and regeneration in experimental chronic pancreatitis. Am. J. Physiol. Gastrointest. Liver Physiol. 282:G1105-G1112.

72. Phillips, P.A., et al. 2003. Rat pancreatic stellate cells secrete matrix metalloproteinases: implications for extracellular matrix turnover. Gut. 52:275-282.

73. Friedman, S.L., and Bansal, M.B. 2006. Reversal of hepatic fibrosis: fact or fantasy? Hepatology. 43(Suppl. 1):S82-S88

74. Perides, G., Tao, X., West, N., Sharma, A., and Steer, M.L. 2005. A mouse model of ethanol dependent pancreatic fibrosis. Gut. 54:1461-1467.

75. Whitcomb, D.C. 2004. Mechanisms of disease: advances in understanding the mechanisms leading to chronic pancreatitis. Nat. Clin. Pract. Gastroenterol. Hepatol. 1:46-52.

76. Masamune, A., Kikuta, K., Satoh, M., Suzuki, N., and Shimosegawa, T. 2005. Protease-activated receptor-2-mediated proliferation and collagen production of rat pancreatic stellate cells. J. Phar macol. Exp. Ther. 312:651-658.

77. Issa, R., et al. 2004. Spontaneous recovery from micronodular cirrhosis: evidence for incomplete resolution associated with matrix cross-linking. Gastroenterology. 126:1795-1808.
78. Jemal, A., et al. 2006. Cancer statistics, 2006. CA Cancer J. Clin. 56:106-130.

79. Mollenhauer, J., Roether, I., and Kern, H.F. 1987. Distribution of extracellular matrix proteins in pancreatic ductal adenocarcinoma and its influence on tumor cell proliferation in vitro. Pancreas. 2:14-24.

80. Armstrong, T., et al. 2004. Type I collagen promotes the malignant phenotype of pancreatic ductal adenocarcinoma. Clin. Cancer Res. 10:7427-7437.

81. Binkley, C.E., et al. 2004. The molecular basis of pancreatic fibrosis: common stromal gene expression in chronic pancreatitis and pancreatic adenocarcinoma. Pancreas. 29:254-263.

82. Koninger, J., et al. 2004. Pancreatic tumor cells influence the composition of the extracellular matrix. Biochem. Biophys. Res. Commun. 322:943-949.

83. Yoshida, S., et al. 2004. Pancreatic cancer stimulates pancreatic stellate cell proliferation and TIMP-1 production through the MAP kinase pathway. Biochem. Biophys. Res. Commun. 323:1241-1245.

84. Bachem, M.G., et al. 2005. Pancreatic carcinoma cells induce fibrosis by stimulating proliferation and matrix synthesis of stellate cells. Gastroenterology. 128:907-921.

85. Watanabe, I., et al. 2003. Advanced pancreatic ductal cancer: fibrotic focus and beta-catenin expression correlate with outcome. Pancreas. 26:326-333.

86. Hartel, M., et al. 2004. Desmoplastic reaction influences pancreatic cancer growth behavior. World J. Surg. 28:818-825.

87. Vaquero, E.C., et al. 2003. Extracellular matrix proteins protect pancreatic cancer cells from death via mitochondrial and nonmitochondrial pathways. Gastroenterology. 125:1188-1202.

88. Edderkaoui, M., et al. 2005. Extracellular matrix stimulates reactive oxygen species production and increases pancreatic cancer cell survival through 5-lipoxygenase and NADPH oxidase. Am. J. Physiol. Gastrointest. Liver Physiol. 289:G1137-G1147.

89. Vaquero, E.C., Edderkaoui, M., Pandol, S.J., Gukovsky, I., and Gukovskaya, A.S. 2004. Reactive oxygen species produced by $\mathrm{NAD}(\mathrm{P}) \mathrm{H}$ oxidase inhibit apoptosis in pancreatic cancer cells. J. Biol. Chem. 279:34643-34654.

90. Munshi, H.G., and Stack, M.S. 2006. Reciprocal interactions between adhesion receptor signaling and MMP regulation. Cancer Metastasis Rev. 25:45-56.

91. Yamamoto, H., et al. 1997. Relation of enhanced secretion of active matrix metalloproteinases with tumor spread in human hepatocellular carcinoma. Gastroenterology. 112:1290-1296.

92. Sternlicht, M.D., and Werb, Z. 2001. How matrix metalloproteinases regulate cell behavior. Annu. Rev. Cell Dev. Biol. 17:463-516.

93. Liotta, L.A., and Kohn, E.C. 2001. The microenvironment of the tumour-host interface. Nature. 411:375-379.

94. Buchholz, M., et al. 2005. Transcriptome analysis of human hepatic and pancreatic stellate cells: organ-specific variations of a common transcriptional phenotype. J. Mol. Med. 83:795-805.

95. Russo, F.P., et al. 2006. The bone marrow functionally contributes to liver fibrosis. Gastroenterology. 130:1807-1821

96. Knittel, T., et al. 1999. Rat liver myofibroblasts and hepatic stellate cells: different cell populations of the fibroblast lineage with fibrogenic potential. Gastroenterology. 117:1205-1221.

97. Knittel, T., et al. 1999. Localization of liver myofibroblasts and hepatic stellate cells in normal and diseased rat livers: distinct roles of (myo-)fibroblast subpopulations in hepatic tissue repair. Histochem. Cell Biol. 112:387-401

98. Uchio, K., et al. 2002. Cellular retinol-binding protein-1 expression and modulation during in vivo and in vitro myofibroblastic differentiation of rat hepatic stellate cells and portal fibroblasts. Lab. 
Invest. 82:619-628.

99. Baba, S., et al. 2004. Commitment of bone marrow cells to hepatic stellate cells in mouse. J. Hepatol. 40:255-260.

100. Forbes, S.J., et al. 2004. A significant proportion of myofibroblasts are of bone marrow origin in human liver fibrosis. Gastroenterology. 126:955-963.

101.Lepreux, S., et al. 2004. Cellular retinol-binding protein-1 expression in normal and fibrotic/cirrhotic human liver: different patterns of expression in hepatic stellate cells and (myo)fibroblast subpopulations. J. Hepatol. 40:774-780.

102. Direkze, N.C., et al. 2004. Bone marrow contribution to tumor-associated myofibroblasts and fibroblasts. Cancer Res. 64:8492-8495.

103.Seaberg, R.M., et al. 2004. Clonal identification of multipotent precursors from adult mouse pancreas that generate neural and pancreatic lineages. Nat. Biotechnol. 22:1115-1124.

104. Horie, Y., and Ishii, H. 2004. What factors play a crucial role in the pathogenesis of alcohol-related chronic pancreatitis and liver cirrhosis? J. Gastroenterol. 39:915-917.

105.Huang, H., et al. 2006. Identification of two gene variants associated with risk of advanced fibrosis in patients with chronic hepatitis C. Gastroenterology. 130:1679-1687.

106.Strnad, P., et al. 2006. Keratin variants associate with progression of fibrosis during chronic hepatitis C infection. Hepatology. 43:1354-1363.

107. Witt, H., et al. 2006. A degradation-sensitive anionic trypsinogen (PRSS2) variant protects against chronic pancreatitis. Nat. Genet. 38:668-673.

108. Yamada, T., et al. 2005. Combination therapy with an angiotensin-converting enzyme inhibitor and an angiotensin II receptor blocker synergistically suppresses chronic pancreatitis in rats. J. Pharmacol. Exp. Ther. 313:36-45.

109.Jaster, R., Sparmann, G., Emmrich, J., and Liebe, S. 2002. Extracellular signal regulated kinases are key mediators of mitogenic signals in rat pancreatic stellate cells. Gut. 51:579-584.

110.McCarroll, J.A., et al. 2004. Pancreatic stellate cell migration: role of the phosphatidylinositol 3-kinase (PI3-kinase) pathway. Biochem. Pharmacol. 67:1215-1225.

111. Masamune, A., Kikuta, K., Satoh, M., Kume, K., and Shimosegawa, T. 2003. Differential roles of signaling pathways for proliferation and migration of rat pancreatic stellate cells. Tohoku J. Exp. Med. 199:69-84.

112. Masamune, A., et al. 2002. Ligands of peroxisome proliferator-activated receptor-gamma block activation of pancreatic stellate cells. J. Biol. Chem. 277:141-147.

113. Yoshida, S., et al. 2005. Pancreatic stellate cells (PSCs) express cyclooxygenase-2 (COX-2) and pancreatic cancer stimulates COX-2 in PSCs. Mol. Cancer. 4:27.

114.McCarroll, J.A., et al. 2006. Vitamin A inhibits pancreatic stellate cell activation: implications for treatment of pancreatic fibrosis. Gut. 55:79-89.

115.Talukdar, R., Saikia, N., Singal, D.K., and Tandon, R. 2006. Chronic pancreatitis: evolving paradigms. Pancreatology. 6:440-449.

116. Dornhofer, N., et al. 2006. Connective tissue growth factor-specific monoclonal antibody therapy inhibits pancreatic tumor growth and metastasis. Cancer Res. 66:5816-5827.

117.Aikawa, T., Gunn, J., Spong, S.M., Klaus, S.J., and
Korc, M. 2006. Connective tissue growth factor-specific antibody attenuates tumor growth, metastasis, and angiogenesis in an orthotopic mouse model of pancreatic cancer. Mol. Cancer Ther. 5:1108-1116.

118.Gomez, J.A., et al. 2004. Vitamin E attenuates biochemical and morphological features associated with development of chronic pancreatitis. Am. J. Physiol. Gastrointest. Liver Physiol. 287:G162-G169.

119.Yoo, B.M., et al. 2005. Novel antioxidant ameliorates the fibrosis and inflammation of ceruleininduced chronic pancreatitis in a mouse model. Pancreatology. 5:165-176.

120.Shimizu, K., et al. 2002. Thiazolidinedione derivatives as novel therapeutic agents to prevent the development of chronic pancreatitis. Pancreas. 24:184-190.

121.Van Westerloo, D.J., et al. 2005. Therapeutic effects of troglitazone in experimental chronic pancreatitis in mice. Am. J. Pathol. 166:721-728.

122. Emori, Y., et al. 2005. Camostat, an oral trypsin inhibitor, reduces pancreatic fibrosis induced by repeated administration of a superoxide dismutase inhibitor in rats. J. Gastroenterol. Hepatol. 20:895-899.

123.Su, S.B., Motoo, Y., Xie, M.J., Taga, H., and Sawabu, N. 2001. Antifibrotic effect of the herbal medicine Saiko-keishi-to (TJ-10) on chronic pancreatitis in the WBN/Kob rat. Pancreas. 22:8-17.

124. Reding, T., et al. 2006. A selective COX-2 inhibitor suppresses chronic pancreatitis in an animal model (WBN/Kob rats): significant reduction of macrophage infiltration and fibrosis. Gut. 55:1165-1173.

125.Yoo, B.M., et al. 2005. Amelioration of pancreatic fibrosis in mice with defective TGF-beta signaling. Pancreas. 30:e71-e79. 